\title{
Telegenetics: The next phase in the provision of genetics services?
}

The article, "Telegenetics in Maine: Successful clinical and educational service delivery model" published in this issue of Genetics in Medicine ${ }^{1}$ demonstrates the continuation of a 30year trend to make genetics services available to remote populations through outreach efforts from clinical genetics units located at tertiary care genetics centers. ${ }^{2,3}$ The need for innovative approaches to the provision of services arises from the increasing awareness of the need for genetics services in many aspects of health care, the continued shortage of genetics professionals, and the economics of the provision of genetics services dictating their primary location in tertiary care centers. With a tradition in the provision of genetics services for a rural hub-and-spoke delivery model, it is only natural that the profession would try telemedicine technology. Although this is not the first report of telegenetics services, it is noteworthy for its multifaceted approach, including the assessment of barriers and attempts to overcome them as part of the evaluation of the program, and for the multipronged evaluation. Rigorous assessment is one key to demonstrating that any new technological advance is safe and acceptable to be used more generally.

Lea et al. ${ }^{1}$ describe a 3-year pilot telegenetics project aiming to increase accessibility to genetics educational and clinical services in Maine. Within this system, genetics service and research units were linked with rural health care centers and public health nurses throughout the state of Maine. During this pilot study, 24 clinical sites participated and 105 patients were evaluated using the videoconferencing infrastructure of the Maine Telemedicine Services. The authors assessed the quality, acceptability, and usefulness of genetics services delivered via telemedicine. Their findings clearly demonstrate the potential of telemedicine technology to increase access to genetics services for rural patients.

Telemedicine is a vehicle for delivering care and not a specific diagnostic or interventional procedure. However, it does alter the process of care delivery and introduces a new communication mode and different social mechanisms into the context of a teleconsultation. The ultimate success of telegenetics will depend on its impact on cost, quality, and access to care.

The cost of telegenetics should be analyzed in relation to how it improves the health of a population by preventing or treating a disease, or increasing knowledge through access to information and communication. The measurement of potential cost savings associated with a telegenetics application depends upon the interest group (e.g., patient, health maintenance organization, provider, or society). Telegenetics applications have the potential to decrease opportunity costs for patients seeking genetics services (by reducing, for instance, travel expenses to visit a specialist). Furthermore, increasing access to health services could lead to increased demand. Emphasis should be also given to the societal perspective, which is most relevant for public policy decisions and encompasses the total costs of resources used to provide a service through telegenetics compared to alternative means of provision of services. In the case of the article in this issue, the cost of telegenetics is a marginal cost because it piggybacks on the Maine Telemedicine Network and is partially covered by a state grant. Therefore, the genetics service does not need to bear the full cost of the ISDN lines and the ITV units, but rather shares these costs, in addition to covering the costs of the line charges used during the visits. This is a beneficial circumstance both for telegenetics and for the larger group using the Maine Telemedicine Network.

When analyzing the quality of care, one needs to investigate diagnostic accuracy, diagnostic impact, and impact of delivered services on patient outcomes. The authors retrospectively assessed the confidence that professionals had in decisions made during a teleconsultation. The next step would be to prospectively compare telegenetics consultations to face-toface consultations; such a study could truly assess if confidence levels of telemedicine consultations were decreased over faceto-face consultations. Given that the majority of telegenetics visits were pediatric diagnostic evaluations, it is reasonable to consider the diagnostic evaluation as a major part of the study. Because most parents would feel anxious about waiting for an appointment at the tertiary care center, they would likely utilize telegenetics if they could get a quicker evaluation. Therefore, it is to be expected that pediatric diagnostic evaluations will continue to be a major component of the Maine telegenetic program.

As the Maine telegenetics program grows, it is probable that many of the visits would be for reasons other than a diagnostic workup. In other telemedicine studies that involve live interaction and not store-and-forward telemedicine, the most popular types of visits tend to be for modalities where studies of communication style and flow are more pertinent than diagnostic accuracy. In genetics, there are many more nondiagnostic encounters such as prenatal and preconception counseling, follow-up by dietitians for patients with metabolic disorders, and cancer genetics counseling where a diagnosis has already been made and the family records have been received ahead of time. In these cases, the evaluation would focus on the actual process of care delivery and communication style and content via videoconferencing rather than diagnostic accuracy. Researchers have developed methods and instruments to explore the nature of communication via telemedicine that could be adapted for use in telegenetics. ${ }^{4}$ Additionally, access to care has 
geographic, social, financial, and psychological components. The development of the telegenetics application described by Lea et al. ${ }^{1}$ aimed to address access barriers to genetics services that rural patients face. Such barriers can be structural or financial (i.e., impediments to genetics services directly related to the concentration of specialists in one area or to the financial cost of the services). In addition, there are personal or cultural barriers that might prevent patients from accessing care services. In a telegenetics application, both patient and provider acceptance of this mode of care delivery is essential to its success. The authors conducted presentations on the telegenetics activities to various physician associations statewide, and continuously and systematically informed the medical community of these new services. These activities ensured that physicians were well informed of the resources available as well as the anticipated benefits associated with their use. Patient satisfaction was also assessed and findings indicate that such a system is perceived as very useful by patients. The patients thought that the best thing about using the telegenetics system was the convenience for the patient and his/her family. Because many genetics visits involve not only the patient but also extended family members, many more family members were able to attend than would have otherwise been possible. Because $64 \%$ of the evaluations were pediatric diagnostic evaluations, the presence of extended family members in many cases is essential for the support of the child's parents.

Telemedicine technology is currently being utilized by most medical specialties (e.g., dermatology, psychiatry, oncology, cardiology, pathology, surgery, and radiology). It is apparent that this technology can also be of use in the domain of genetics. As is the case with every information system, the systematic study and evaluation of telegenetics systems will provide insight into factors of successful design, implementation, and best practices. As demonstrated by the article in this issue, telegenetics has great promise; however, the authors are careful to evaluate the factors necessary to make their telegenetics ventures a success and not just assume that it will be successful in genetics because it is used by other specialties. The authors used a multifaceted approach to establishing telegenetics services, including specific measures to address four barriers that have been discovered by other telemedicine programs and often the reasons for underutilization of telemedicine services. These four barriers include (1) lack of knowledge of health care workers and patients about telemedicine services, (2) lack of understanding by health care workers and patients about the role of genetics services in patient care, (3) logistical issues of where the ITV unit was located and how it was scheduled or moved, and (4) issues of hospital credentialing and privileges so that medical services could be provided by the geneticists at sites outside their home institution. Reimbursement for services would have been a barrier in prior years, but the authors' program was able to obtain reimbursement from Maine Medicaid. We encourage more studies and more articles to clearly demonstrate that the use of telemedicine services in genetics is cost effective, safe, diagnostically acceptable, and also acceptable to providers and patients and their families. For this pur- pose, we urge the utilization of evaluation instruments and frameworks already developed for the use of telemedicine in other clinical specialties, such as those outlined in the general guide to evaluating telemedicine applications developed by the Institute of Medicine. ${ }^{5}$

In the era of postgenomic medicine, the provision of genetics testing, counseling, and associated services is becoming a critical part of many patient encounters. ${ }^{6}$ Innovation in the profession related to the delivery of services, especially with the utilization of distance technologies such as telemedicine, will be essential to providing genetics services to the population. Telemedicine is one of the dimensions of "e-health," defined broadly as the use of advanced information technologies to improve health care delivery and education. This concept refers to a fundamental redesign of health care processes based on the use and integration of electronic communication at all levels. Telemedicine services will likely progress to include Internet-enabled consultations, leading to more geographic freedom for the patients instead of being restricted to the fixed set of sites within a specific delivery network. E-health leads to patient empowerment, which describes the transition from a passive role where the patient is the recipient of care services to an active role where the patient is informed, has choices, and is involved in the decision-making process. Web sites for consumers, such as the Genetics Home Reference, ${ }^{7}$ are also a valuable part of the genetics e-health armamentarium. As genetics becomes an essential part of health care delivery, the use of technology can ensure access to such services for a greater number of people and increase understanding of the domain. With the great tradition in the field of medical genetics for innovation in services provision, surely the use of telegenetics will become widely diffused. The challenge for researchers, health care organizations, and policy-makers lies in extensively evaluating telegenetics technologies, properly adopting them, and making informed decisions about their appropriate use.

Joyce A. Mitchell, PhD

George Demiris, PhD

Department of Health Management and Informatics

University of Missouri

Columbia, Missouri

\section{References}

1. Lea DH, Johnson JL, Ellingwood S, Allman W, Patel A, Smith R. Telegenetics in Maine: successful clinical and educational service delivery model developed from a three year pilot project. Genet Med 2005;7:21-27.

2. Epstein CJ, Erickson RP, Hall BD, Golbus MS. The center-satellite system for the wide-scale distribution of genetic counseling services. Am J Hum Genet 1975;27:322332.

3. Riccardi VM. Health care and disease prevention through genetic counseling: regional approach. Am J Public Health 1976;66:268-272.

4. Demiris G, Speedie S, Finkelstein S, Harris I. Communication patterns and technical quality of virtual visits in home care. J Telemed Telecare 2003;9:210-215.

5. Field MJ, ed. Telemedicine: A guide to assessing telecommunications in health care Washington, DC: U.S. Institute of Medicine: Committee on Evaluating Clinical Applications of Telemedicine; 1996.

6. Epstein CJ. Genetic testing: hope or hype? Genet Med 2004;6:165-172.

7. Mitchell JA, Fun J, McCray AT. Design of Genetics Home Reference: a new NLM consumer health resource. J Am Med Inform Assoc 2004;11:439-447. 